\title{
Attention Deficit-Hyperactivity Disorder (ADHD)
}

National Institute of Neurological Disorders and Stroke (NINDS)

\section{Source}

National Institute of Neurological Disorders and Stroke (NINDS). Attention DeficitHyperactivity Disorder Information Page.

Attention deficit-hyperactivity disorder (ADHD) is a neurobehavioral disorder that affects 3-5 percent of all American children. It interferes with a person's ability to stay on a task and to exercise age-appropriate inhibition (cognitive alone or both cognitive and behavioral). Some of the warning signs of ADHD include failure to listen to instructions, inability to organize oneself and school work, fidgeting with hands and feet, talking too much, leaving projects, chores and homework unfinished, and having trouble paying attention to and responding to details. There are several types of ADHD: a predominantly inattentive subtype, a predominantly hyperactive-impulsive subtype, and a combined subtype. ADHD is usually diagnosed in childhood, although the condition can continue into the adult years. 